\title{
Role of Platelet-Rich Plasma in Articular Cartilage Injury and Disease
}

\author{
Randy Mascarenhas, MD, FRCSC ${ }^{1}$ Bryan M. Saltzman, MD ${ }^{1}$ Lisa A. Fortier, PhD, DVM ${ }^{2}$ \\ Brian J. Cole, MD, MBA ${ }^{1}$ \\ ${ }^{1}$ Department of Orthopedic Surgery, Rush University Medical Center, \\ Chicago, Illinois \\ ${ }^{2}$ Cornell School of Veterinary Medicine, Ithaca, New York \\ Address for correspondence Brian J. Cole, MD, MBA, Department of \\ Orthopedic Surgery, Rush University Medical Center, 1611 West \\ Harrison Street, Suite 300, Chicago, IL 60612 \\ (e-mail: brian.cole@rushortho.com).
}

J Knee Surg 2015;28:3-10.

\begin{abstract}
Keywords

- articular cartilage

- osteoarthritis

- platelet-rich plasma

- PRP

Clinical and laboratory research aimed at biological approaches to cartilage repair are currently in high demand due to the poor regenerative capacity of articular cartilage in the setting of a diseased articular environment. Platelet-rich plasma (PRP) takes advantage of supraphysiological concentrations of platelets and their growth factors harbored in $\alpha$-granules, which together attempt to return the diseased articular cartilage to a preinjury state. The local use of PRP directly at the site of cartilage injury is thought to stimulate a natural healing cascade and accelerate the formation of cartilage repair tissue. This article provides an overview of the basic science behind the use of PRP in the treatment of cartilage injury and disease. Both initial and current examples of the use of intra-articular PRP in clinical human studies are provided. These include the use of PRP either alone or as an augmentation device with various other procedures, including arthroscopic microfracture and cell-free resorbable polyglycolic acid-hyaluronan implantation. Finally, the authors describe some of the potential future roles of PRP in clinical settings based on recent literature. These include Achilles tendon rupture, chronic tendinosis, chronic rotator cuff tendinopathy or tearing, muscle injury, and meniscal repair.
\end{abstract}

The articular cartilage in the setting of a diseased articular environment has very poor regenerative capacity. Thus, clinical and laboratory research aimed at biological approaches to repair cartilage injury using growth factors provides promise for the treatment of disabling articular cartilage disease. Growth factors are naturally occurring substances-often proteins or steroid hormones-that are capable of stimulating cellular differentiation, growth or proliferation while serving an important role in regulating various cellular processes. Numerous growth factors have quantitative and temporal effects on articular cartilage growth including transforming growth factor- $\beta 1$ (TGF- $\beta 1$ ), bone morphogenetic protein- 2 and -7 , insulin growth factor1 , fibroblast growth factor- 2 and -18 (FGF-2, FGF-18), vascular endothelial growth factor (VEGF), epidermal growth factor (EGF), and platelet-derived growth factor (PDGF). Many of these factors are found stored in the $\alpha$-granules of platelets, including VEGF, TGF- $\beta$, EGF, FGF, and PDGF. Independently, these factors serve to promote local angiogenesis, modulate inflammation, inhibit catabolic enzymes and cytokines, recruit local stem cells and fibroblasts to sites of damage or injury, and induce healthy nearby cells to manufacture greater numbers of growth factors. ${ }^{1}$ In combination, these proanabolic and anticatabolic effects attempt to return injured or diseased articular cartilage to its preinjury state. Platelet-rich plasma (PRP) is a sample of plasma with a supraphysiological concentration of platelets manufactured to harness and unleash these anabolic effects on injured or diseased cartilage in received

April 1, 2014

accepted after revision

June 4, 2014

published online

July 28, 2014
Copyright $\odot 2015$ by Thieme Medical Publishers, Inc., 333 Seventh Avenue, New York, NY 10001, USA. Tel: +1(212) 584-4662.
DOI http://dx.doi.org/ 10.1055/s-0034-1384672. ISSN 1538-8506. 
an effort to augment cartilage regeneration. Platelets and their associated cytokines and growth factors comprise the workhorse of the biological mixture, although the plasma contains valuable biological molecules also involved in injury repair. $^{2}$

\section{Basic Science behind Platelet-Rich Plasma}

Osteoarthritis is a chronic degenerative joint disease in which the catabolic activity that becomes favored within chondrocytes leads to eventual articular cartilage wear. ${ }^{2}$ Cartilage has an extremely limited ability for self-repair given its avascularity $^{3}$; thus, the traditional inflammatory repair process does not contribute to the healing response in the setting of cartilage injury as it has no means to travel to the locally affected tissue. The rationale for the use of PRP is that the supraphysiological release of platelet-derived factors at the direct site of cartilage injury or disease can stimulate the natural healing cascade and tissue regeneration. ${ }^{4}$ Platelet activation leads to a release of the aforementioned growth factors and hundreds of others from its $\alpha$-granules to promote cartilage matrix synthesis, increase cell growth, migration, and phenotype changes, and facilitate protein transcription within chondrocytes. ${ }^{5,6}$ The chemoattractants stored in platelets draw proteins, such as fibrinogen and fibrin, the latter of which acts as an initial scaffold for stem cells to migrate and differentiate. Generally, basic science evidence has demonstrated the ability of PRP to increase mesenchymal stem cell and chondrocyte proliferation, deposit type II collagen and proteoglycan. ${ }^{7,8}$ This in theory may accelerate the formation of cartilage repair tissue.

The abundance of platelets in PRP increases the concentrations of relevant substances locally leading to a sustained effect on articular cartilage. The transcription of many degradative cytokines including interleukin-1 $\beta$, tumor necrosis factor- $\alpha$, and interleukin- 6 are under the upstream control of nuclear factor KB (NF-KB), and the $\alpha$-granule contents in platelets inhibit this catabolic pathway on the downstream end and prevent the otherwise detrimental effects on articular cartilage changes in the process of osteoarthritis. ${ }^{9-11}$ Activated PRP increases in vitro levels of hepatocyte growth factor, which enhances cellular $\mathrm{IkB} \alpha$ expression and subsequently disrupts the NF-KB transactivating activity. It does so via NF-KB-p65 subunit cytosolic retention and nucleocytoplasmic shunting, thus decreasing its downstream proinflammatory effects. ${ }^{12}$ PRP additionally has antinociceptive and anti-inflammatory properties, which result from the ability of PRP to decrease synoviocyte matrix metalloproteinase-13 expression as shown in cartilage explant studies, which would otherwise have a primary role in cartilage matrix degradation while undergoing osteoarthritic changes. The same research demonstrated significantly increased hyaluronan synthase-2 expression in PRP-treated samples, which is an enzyme known to produce large hyaluronic acid (HA) isoforms and thus contribute to the cartilage construct (-Fig. 1). ${ }^{11}$ Of final note, PRP decreases the expression of cyclooxygenase-2 and chemokine-receptor CXCR4 target genes which may regulate local inflammation when used in the setting of articular cartilage injury. ${ }^{12}$

\section{Platelet-Rich Plasma in the Treatment of Articular Cartilage Injury}

Preclinical animal studies on PRP have appraised its utility in both in vivo and in vitro repair of focal cartilage lesions and

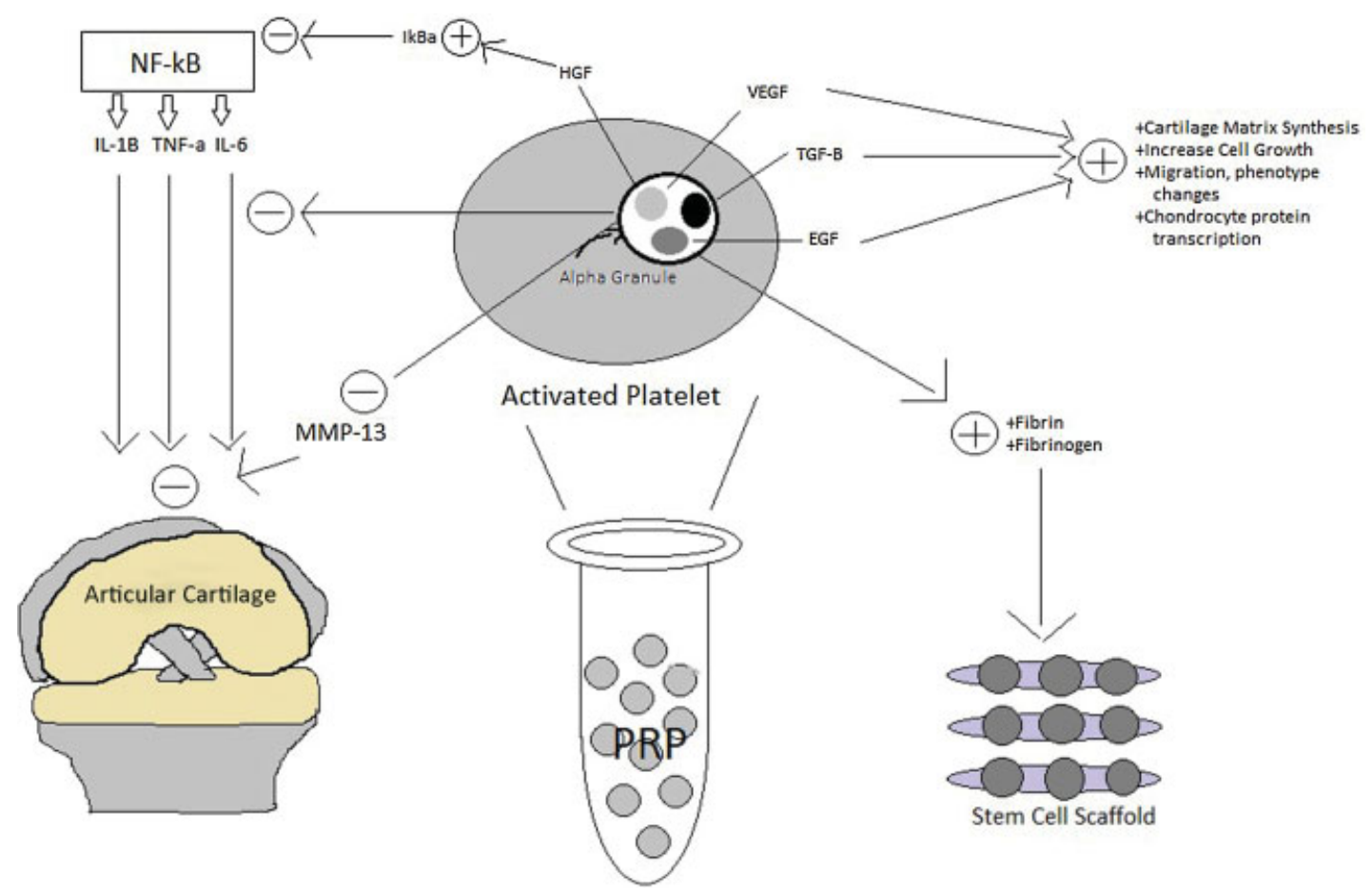

Fig. 1 The PRP activation pathway. EGF, epidermal growth factor; HGF, hepatocyte growth factor; IL, interleukin; MMP, matrix metalloproteinase; PRP, platelet-rich plasma; TGF, transforming growth factor; TNF, tumor necrosis factor; VEGF, vascular endothelial growth factor. 
osteoarthritis therapy. ${ }^{5}$ Many of the early clinical human studies have reported on PRP use in the treatment of osteoarthritis, with specific focus on the ability of PRP to achieve patient-reported improvements in pain and symptoms during the treatment of such articular cartilage pathology ( - Table 1).

A randomized double-blinded study of 78 total patients by Patel et $\mathrm{al}^{13}$ demonstrated that PRP injections into the knee of patients with mild or moderate osteoarthritis produced higher Western Ontario and McMaster Universities Arthritis (WOMAC) subjective knee symptom scores when compared with a saline injection control cohort. Filardo et al ${ }^{14}$ prospectively evaluated 91 patients who received three PRP injections every 3 weeks for degenerative osteoarthritic knee chondral lesions and reported a median duration of improvement of 9 months. Outcome measures were still improved from baseline at 2 years but were not maintained at the same level as the 1-year level of improvement, thus leading the authors to question the long-term efficacy of PRP. The research team also determined that superior results were gathered from younger patients and those with less cartilage degradation. Kon et al ${ }^{15}$ prospectively followed 100 patients with degenerative chondral lesions and osteoarthritis who received three PRP injections every 3 weeks, and similarly reported superior responses to treatment in younger patients and a decrease in the improvement of outcomes at 1 year following injections when compared with the results at 6 months. Sampson et al ${ }^{16}$ prospectively followed 14 patients with knee osteoarthritis treated with three PRP injections at 4-week intervals and reported significant improvements in pain and symptom relief in the majority of patients at 12 months postinjection as per Knee Injury and Osteoarthritis Outcome Score (KOOS) and Brittberg-Peterson visual analog scale (VAS) scores.

Filardo et $\mathrm{al}^{17}$ compared PRP to HA injections in the treatment of knee chondropathy or osteoarthritis and reported a trend toward favorable outcomes in the PRP group at 1-year follow-up for patients with low-grade articular degeneration, but no superiority to HA injections in middleaged patients with moderate signs of arthritis. By contrast, significantly better results in WOMAC index and Numeric Rating Scale scores were recorded in patients receiving three PRP injections as compared with HA injections for knee osteoarthritis at 3- and 6-month follow-up, as per the findings of Spaková et al. ${ }^{18}$

Gobbi et $\mathrm{al}^{4}$ treated 50 patients with knee osteoarthritis using two intra-articular injections of autologous PRP and reported significant improvements in all outcome scoring scales at both 6 - and 12 -month follow-up with $100 \%$ return to previous activities. These results were irrespective of whether or not the patients had undergone previous operative intervention for cartilage lesions (cartilage shaving and/or microfracture). Total 50 consecutive patients with grade II or III chondromalacia underwent 1 year of treatment with nine PRP injections by Hart et al, ${ }^{19}$ with the results demonstrating significant improvements in all measured outcome scores. Magnetic resonance imaging determined that despite the reduced pain and improved quality of life in these patients, there was no significant cartilage regeneration. Jang et $\mathrm{al}^{20}$ prospectively evaluated 65 patients suffering from osteoarthritis treated with intra-articular PRP injection; their results showed statistically significant improvements in several outcome scores, but pain relapsed at an average 8.8 months after the procedure. Increased age and the presence of patellofemoral joint degeneration worsened the outcomes with PRP in this study. Similarly, Kon et $\mathrm{al}^{21}$ reported on 50 patients with degenerative cartilage lesions of the knee and severe osteoarthritis who were treated with three autologous PRP intraarticular injections and found that PRP had longer therapeutic efficacy than HA injections in comparable demographic cohorts. As with many of the aforementioned clinical studies, superior results were reported in younger and more active patients with a lower degree of cartilage degeneration.

The variety of techniques for PRP production has also been compared in patients with degenerative knee cartilage lesions and osteoarthritis. Filardo et $\mathrm{al}^{22}$ compared 72 patients treated with three injections of PRP prepared with a singlespinning procedure (plasma rich in growth factors [PRGF]) to an equal number of patients treated in similar fashion with PRP prepared with a double-spinning approach. The authors reported statistically significant improvements in subjective knee clinical outcome scores at 2-, 6-, and 12-month followup, particularly in younger patients with a lower degree of cartilage degeneration. There were no differences in the comparative analysis of the two groups at these follow-up outcome time points, although a significantly larger number of double-spinning PRP injections produced transient local pain and swelling reactions.

While most clinical studies on PRP have evaluated its use in patients with chronic degenerative cartilage disease, a single case report from Freitag et $\mathrm{al}^{23}$ found good efficacy for a course of photoactivated PRP injections in a 38-year-old patient with a traumatic focal chondral lesion of the knee from a basketball injury. Another case reported by Sánchez et $\mathrm{al}^{24}$ reported accelerated articular cartilage healing and excellent symptomatic improvement in a patient with a nontraumatic knee cartilage avulsion injury treated by arthroscopic reattachment supplemented with PRP injection.

PRP has recently been studied as an augmentation device with various other cartilage procedures for the purpose of osteochondral lesion treatment with early reports of success (-Table 2). Guney et $\mathrm{al}^{25}$ evaluated treatment for osteochondral lesions of the talus by comparing arthroscopic microfracture alone to that augmented with PRP injection on the 1st postoperative day. The latter cohort of patients had significantly superior American Orthopaedic Foot and Ankle Society (AOFAS) scores, Foot and Ankle Ability Measure (FAAM) overall pain domain and 15-minute walking domain subgroup scores, and VAS pain scores in comparison to isolated arthroscopic microfracture. Siclari et $\mathrm{al}^{26}$ reported significant KOOS improvements in all subcategories after treatment of focal knee chondral defects with a cell-free resorbable polyglycolic acid-hyaluronan implant immersed with autologous PRP after bone marrow stimulation via subchondral drilling. The improvement seen at 1 year postoperatively was still present at 2 years after the surgery, with 


\begin{tabular}{|c|c|c|c|c|c|c|c|}
\hline 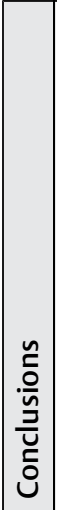 & 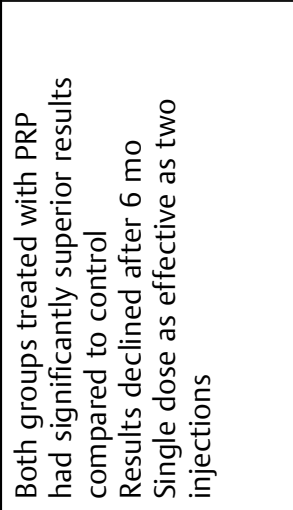 & 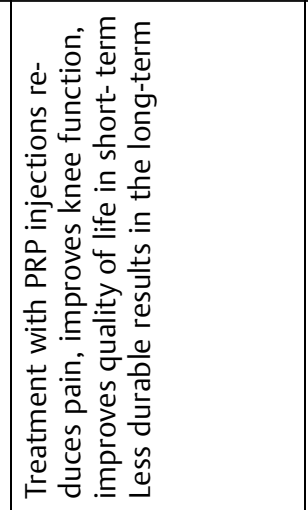 & 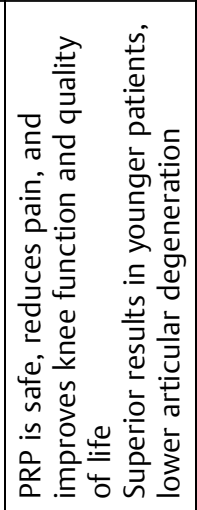 & 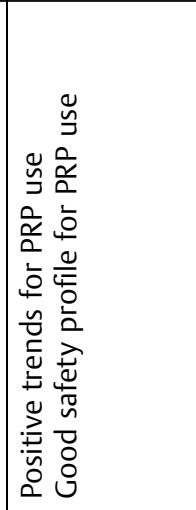 & 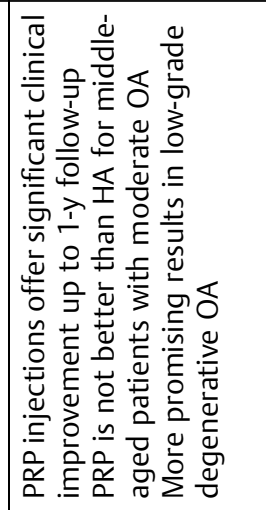 & 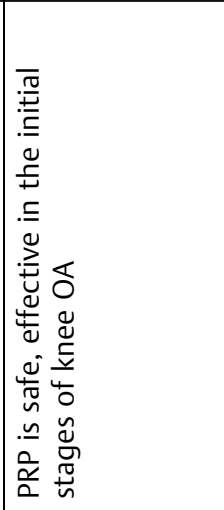 & 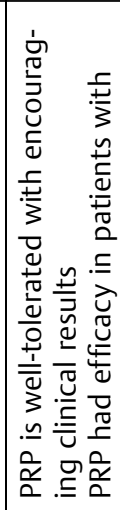 \\
\hline $\mid \begin{array}{c}\frac{n}{z} \\
\vec{z} \\
\check{g}\end{array}$ & 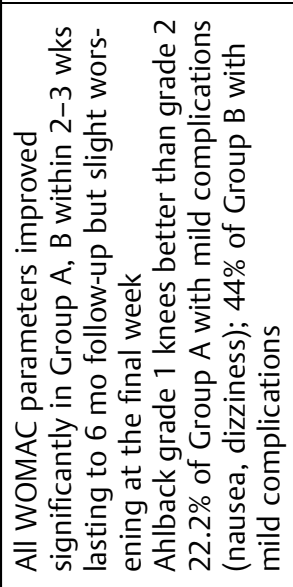 & 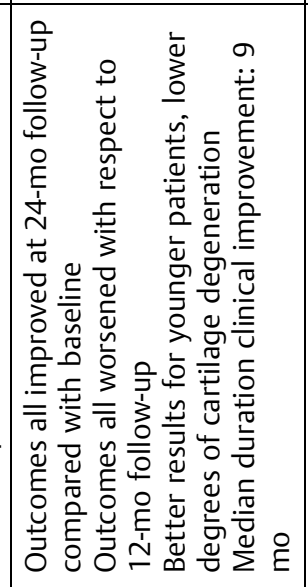 & 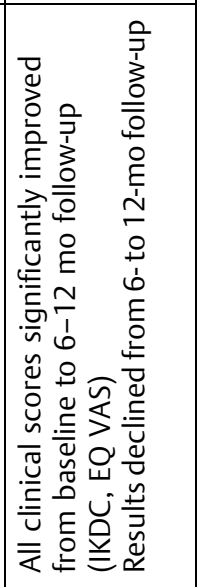 & 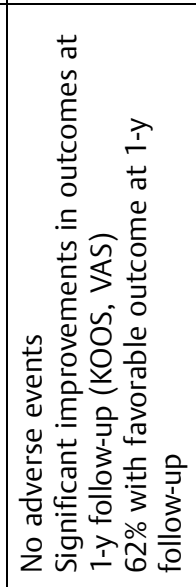 & 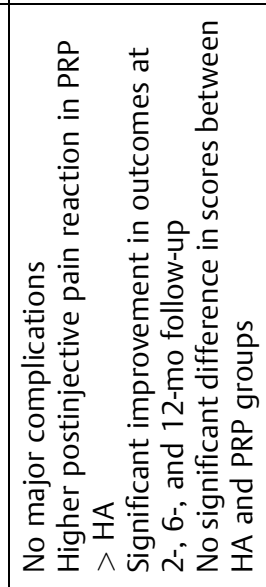 & 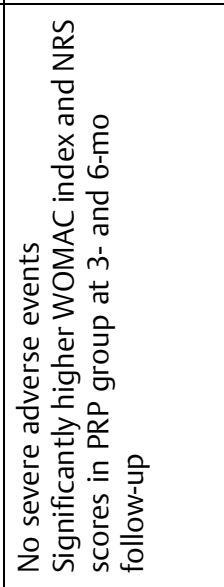 & 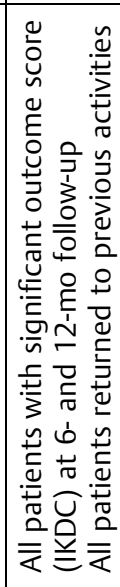 \\
\hline 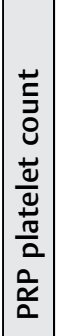 & 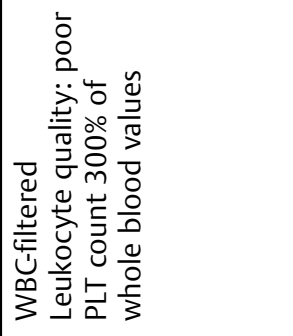 & 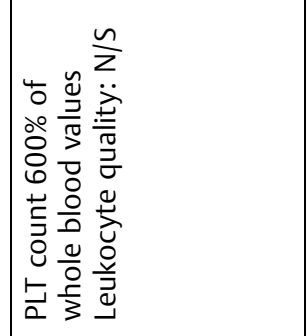 & 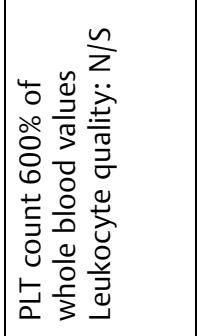 & 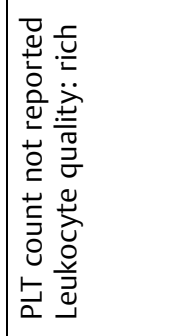 & 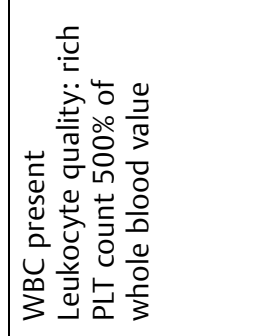 & 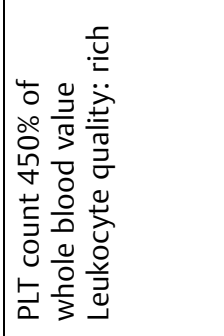 & 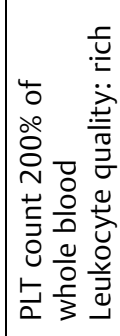 \\
\hline $\mid \begin{array}{l}n \\
\tilde{D} \\
\frac{1}{ \pm} \\
\tilde{u} \\
\Sigma\end{array}$ & 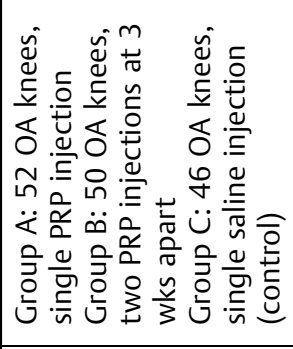 & 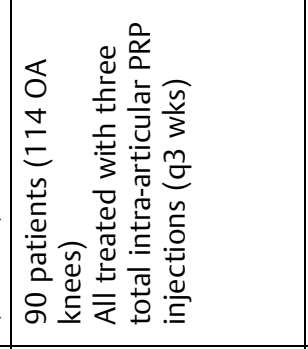 & 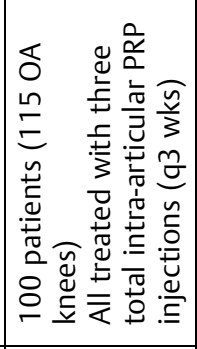 & 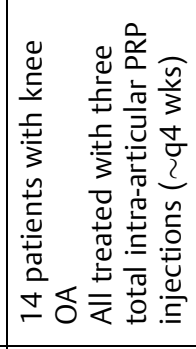 & 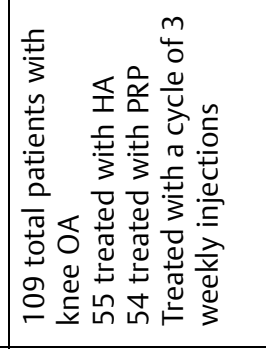 & 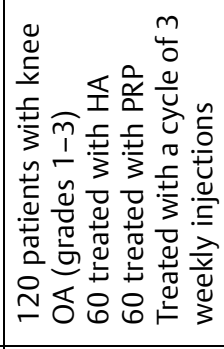 & 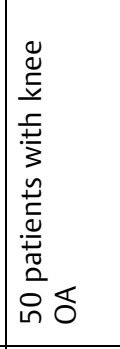 \\
\hline 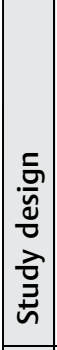 & 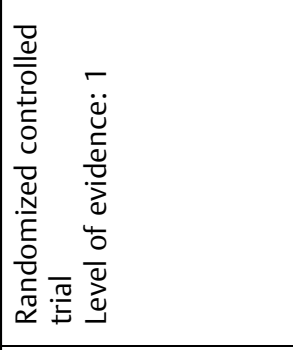 & 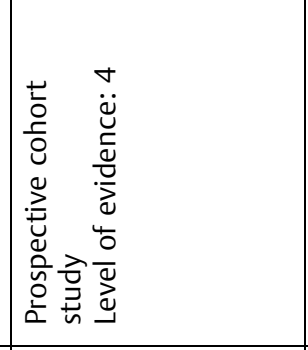 & 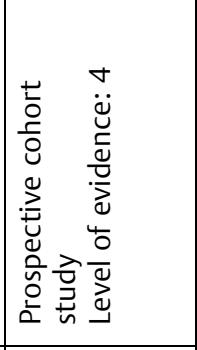 & 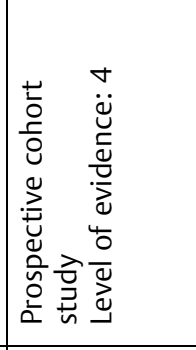 & 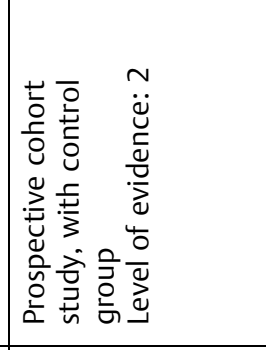 & 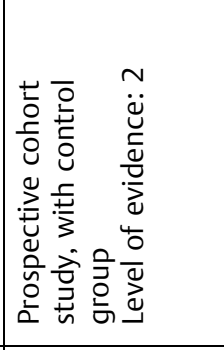 & 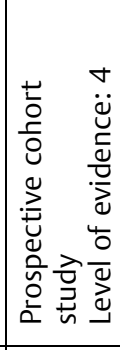 \\
\hline 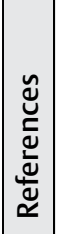 & 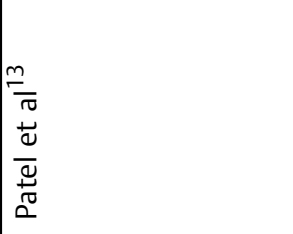 & 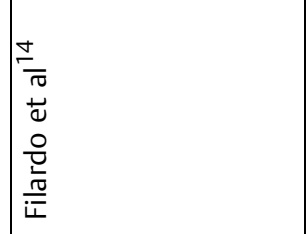 & $\mid \begin{array}{l}\bar{\sigma} \\
+ \\
\tilde{\alpha} \\
\bar{\sigma} \\
\underline{v}\end{array}$ & 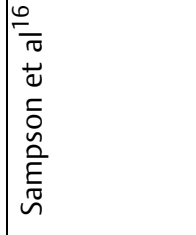 & 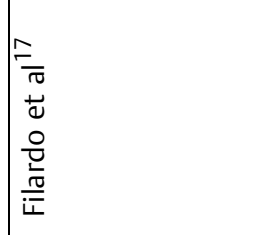 & 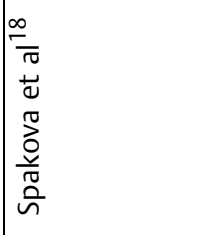 & $\begin{array}{l}\bar{T} \\
\overline{0} \\
-\overline{0} \\
\overline{0} \\
0\end{array}$ \\
\hline
\end{tabular}



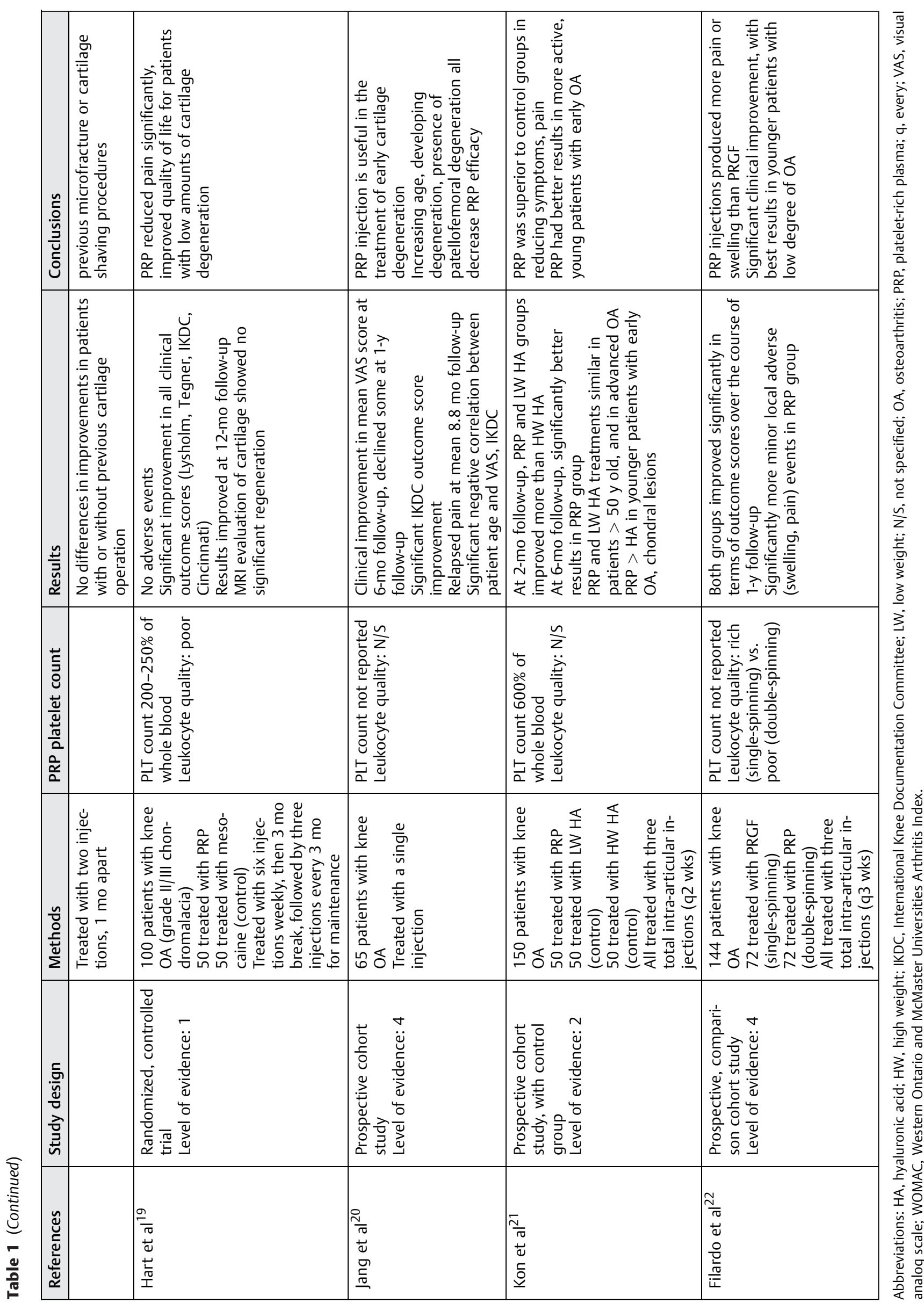


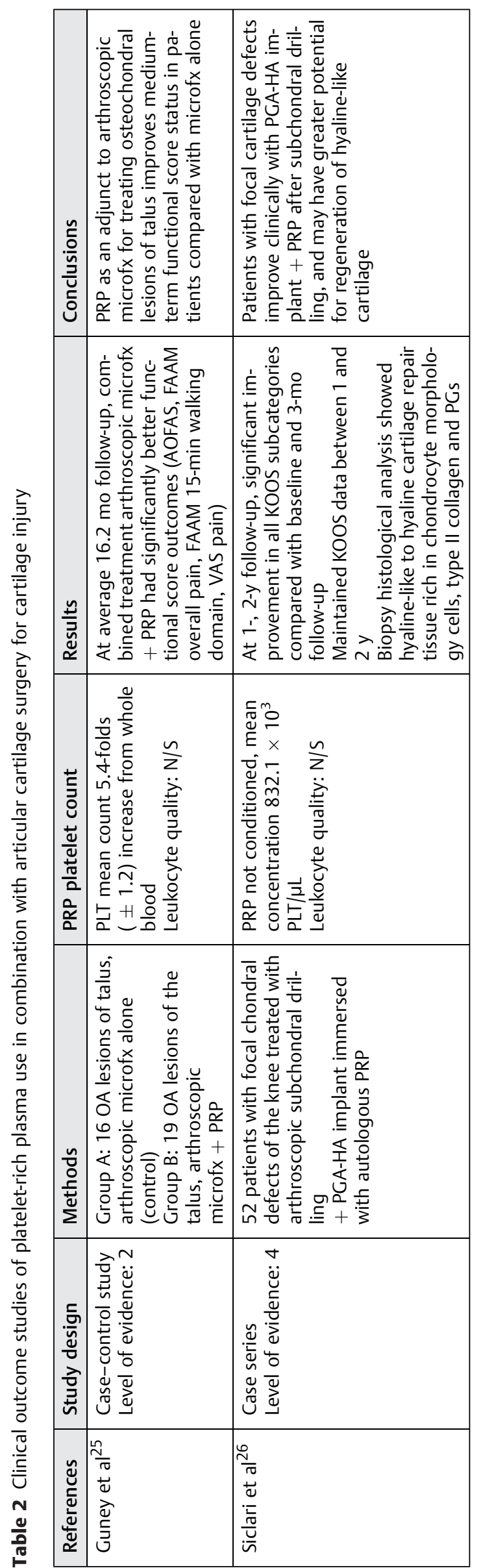

histological analysis of biopsy tissue showing potential regeneration of hyaline-like cartilage. Recently, the senior author (B.J.C.) published a promising technique using micronized allogeneic articular cartilage combined with PRP as a scaffold and adjunct to traditional microfracture surgery (BioCartilage, Arthrex, Inc., Naples, FL). ${ }^{27}$

\section{Future Roles for Platelet-Rich Plasma in Treating Cartilage Disease}

Cartilage damage can take place secondary to both acute and chronic processes. Acute traumatic injury to articular cartilage can create focal chondral loss and potentially lead to a more widespread osteoarticular problem. By contrast, chronic joint stress over a prolonged period of time may lead to eventual widespread inflammatory chondral degradation and osteoarthritis. Most studies evaluating PRP have evaluated its use within the setting of uniform cartilage degeneration as seen in osteoarthritis. While relatively devoid from the literature in human clinical studies, animal studies have evaluated the proposed effect of PRP on focal osteochondral defects (-Fig. 2). Sun et al $^{3}$ evaluated the treatment of large osteochondral defects created in the patellofemoral groove of rabbit models with treatment arms consisting of PRP in a polylactic-glycolic acid (PLGA), PLGA alone, or no treatment. The authors reported no significant differences between the PRP and untreated groups in terms of macroscopic examination, microcomputed tomography (larger amount of subchondral bone formation) and histological changes of the newly formed bone and cartilage within the defect after 12 weeks. With the knowledge of the efficacy of PRP in younger cohorts, future clinical evaluations of the use of PRP with younger patients sustaining acute osteochondral defects would be valuable. ${ }^{23}$ Moreover, the utility of PRP in the treatment of cartilage degeneration and injury in joints other than the knee is largely unreported and may become a worthwhile research venue that is pursued.

Aside from cartilage degeneration, the clinical use of PRP has been studied in numerous clinical and preclinical reports to determine value in the following sports medicine pathologies: Achilles tendon rupture, chronic tendinosis, chronic rotator cuff tendinopathy or tearing, muscle injury, and meniscal repair. ${ }^{28}$ Sadoghi et al $^{29}$ systematically reviewed the use of PRP in the treatment of Achilles tendon ruptures in animal and human studies, and found that analysis of 14 total studies demonstrated significant effects of PRP in the treatment of Achilles tendon ruptures likely secondary to enhanced scar tissue maturation. Krogh et $\mathrm{al}^{30}$ systematically reviewed and analyzed randomized controlled trials of injection therapies for patients with lateral epicondylitis, and determined that PRP was statistically superior to placebo, although most studies were at risk for bias using the Cochrane risk of bias tool. Carter et al $^{31}$ analyzed 24 articles evaluating PRP use in advanced wound therapy and concluded that PRP therapy in cutaneous wounds showed improved partial and complete wound healing compared with wound care alone in a control group. Villela et $\mathrm{al}^{32}$ reported similar conclusions in the treatment of diabetic ulcers with PRP. These additional 

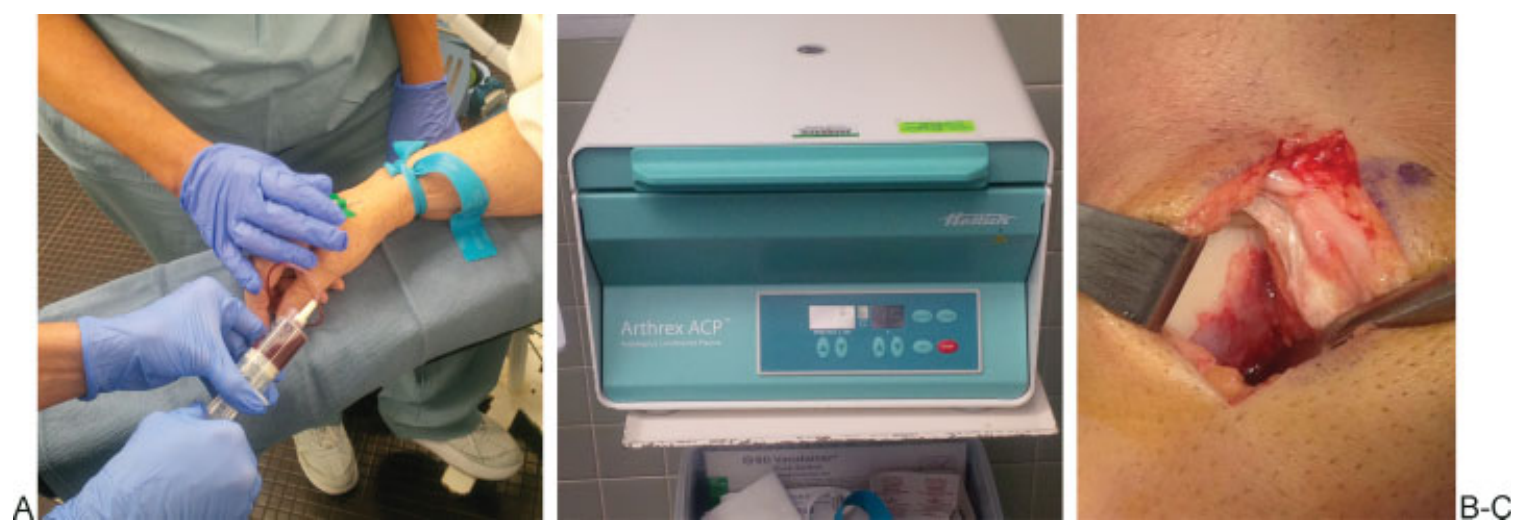

Fig. 2 (A) A blood draw is performed on the patient intraoperatively after the induction of anesthesia. (B) The blood is spun down in a centrifuge to separate out the platelet-rich plasma. (C) The platelet-rich plasma is placed at the microfracture site with a fibrin glue sealant.

uses for PRP continue to be studied with higher levels of evidence, with the hope that more definitive conclusions can be made either in favor of or against routine use in the orthopedics.

While most of the cartilage-related research on the efficacy of PRP has been devoted to its effect on chondrocytes thus far, recent studies have examined the effect of both leukocyte-rich and leukocyte-poor PRP on synoviocytes. More than $80 \%$ of normal human synovium is composed of synoviocytes that produce cytokines and matrix metalloproteinases that can mediate cartilage metabolism. While no differences in clinical outcomes have been observed till date between leukocyte-rich and leukocyte-poor derivatives, a recent laboratory study by Braun et $\mathrm{al}^{33}$ found that leukocyte-rich PRP and red blood cell concentrates both led to significantly greater cell death and proinflammatory mediator production than leukocyte-poor PRP or plateletpoor plasma. These findings led the authors to suggest that clinicians consider using leukocyte-poor, red blood cell-free formulations of PRP when performing intra-articular procedures. Further research and clinical correlation is required in this area to determine whether clinical consequences may exist secondary to the release of catabolic proteases that may perpetuate inflammation and potentially inhibit tissue healing.

\section{Conclusion}

There are numerous basic science and clinical studies demonstrating the positive effects that PRP has on cartilage degeneration or injury, with many of the aforementioned in vivo studies exhibiting improvements in both symptoms and joint function. The improvement in symptoms after intra-articular injection of PRP in knee osteoarthritis is short-term, with many studies demonstrating declines in efficacy after 1 year. In addition, both age and degree of cartilage degeneration have an inverse relationship with the effectiveness of PRP. PRP as a treatment option for cartilage damage or injury is attractive given the low rate of adverse events observed in numerous clinical studies, its simplicity of quickly obtaining a sample of autogenous whole blood, and the absent risk of disease transmission. PRP has been used as an adjunct to several cartilage-related procedures including microfracture surgery and graft, scaffold and implant insertion. ${ }^{7}$ Ultimately, however, recent systematic reviews on the topic conclude that there is still a paucity of high-quality data providing sufficient evidence to support or disprove the clinical utility of PRP in symptomatic osteoarthritis of the knee. ${ }^{34}$ There is even less clinical evidence supporting its use in other joints or in the treatment of focal osteochondral defects despite the basic science evidence in favor of its use. ${ }^{35}$ In addition, not all basic science and clinical studies on PRP have concluded it has positive effects. ${ }^{7}$ Further studiesparticularly randomized, controlled trails-to evaluate the in vivo effects of PRP on human cartilage are imperative to conclusively determine the proper patient population and expected outcomes for the use of PRP in the setting of cartilage damage and injury.

\section{References}

1 Fortier LA, Barker JU, Strauss EJ, McCarrel TM, Cole BJ. The role of growth factors in cartilage repair. Clin Orthop Relat Res 2011; 469(10):2706-2715

2 Zhu Y, Yuan M, Meng HY, et al. Basic science and clinical application of platelet-rich plasma for cartilage defects and osteoarthritis: a review. Osteoarthritis Cartilage 2013;21(11):1627-1637

3 Sun Y, Feng Y, Zhang CQ, Chen SB, Cheng XG. The regenerative effect of platelet-rich plasma on healing in large osteochondral defects. Int Orthop 2010;34(4):589-597(SICOT)

4 Gobbi A, Karnatzikos G, Mahajan V, Malchira S. Platelet-rich plasma treatment in symptomatic patients with knee osteoarthritis: preliminary results in a group of active patients. Sports Health 2012;4(2):162-172

5 Fortier LA, Hackett CH, Cole BJ. The effects of platelet-rich plasma on cartilage: basic science and clinical application. Oper Tech Sports Med 2011;19:154-159

6 Weiser L, Bhargava M, Attia E, Torzilli PA. Effect of serum and platelet-derived growth factor on chondrocytes grown in collagen gels. Tissue Eng 1999;5(6):533-544

7 Smyth NA, Murawski CD, Fortier LA, Cole BJ, Kennedy JG. Plateletrich plasma in the pathologic processes of cartilage: review of basic science evidence. Arthroscopy 2013;29(8):1399-1409

8 Krüger JP, Hondke S, Endres M, Pruss A, Siclari A, Kaps C. Human platelet-rich plasma stimulates migration and chondrogenic 
differentiation of human subchondral progenitor cells. J Orthop Res 2012;30(6):845-852

9 Abrams GD, Frank RM, Fortier LA, Cole BJ. Platelet-rich plasma for articular cartilage repair. Sports Med Arthrosc 2013;21(4): 213-219

10 Hayden MS, Ghosh S. Shared principles in NF-kappaB signaling. Cell 2008;132(3):344-362

11 Sundman EA, Cole BJ, Karas V, et al. The anti-inflammatory and matrix restorative mechanics of platelet-rich plasma in osteoarthritis. Am J Sports Med 2014;42(1):35-41

12 Bendinelli P, Matteucci E, Dogliotti G, et al. Molecular basis of antiinflammatory action of platelet-rich plasma on human chondrocytes: mechanisms of NF-KB inhibition via HGF. J Cell Physiol 2010; 225(3):757-766

13 Patel S, Dhillon MS, Aggarwal S, Marwaha N, Jain A. Treatment with platelet-rich plasma is more effective than placebo for knee osteoarthritis: a prospective, double-blind, randomized trial. Am J Sports Med 2013;41(2):356-364

14 Filardo G, Kon E, Buda R, et al. Platelet-rich plasma intra-articular knee injections for the treatment of degenerative cartilage lesions and osteoarthritis. Knee Surg Sports Traumatol Arthrosc 2011; 19(4):528-535

15 Kon E, Buda R, Filardo G, et al. Platelet-rich plasma: intra-articular knee injections produced favorable results on degenerative cartilage lesions. Knee Surg Sports Traumatol Arthrosc 2010;18(4): 472-479

16 Sampson S, Reed M, Silvers H, Meng M, Mandelbaum B. Injection of platelet-rich plasma in patients with primary and secondary knee osteoarthritis: a pilot study. Am J Phys Med Rehabil 2010;89(12): 961-969

17 Filardo G, Kon E, Di Martino A, et al. Platelet-rich plasma vs hyaluronic acid to treat knee degenerative pathology: study design and preliminary results of a randomized controlled trial. BMC Musculoskelet Disord 2012;13:229-236

18 Spaková T, Rosocha J, Lacko M, Harvanová D, Gharaibeh A. Treatment of knee joint osteoarthritis with autologous platelet-rich plasma in comparison with hyaluronic acid. Am J Phys Med Rehabil 2012;91(5):411-417

19 Hart R, Safi A, Komzák M, Jajtner P, Puskeiler M, Hartová P. Plateletrich plasma in patients with tibiofemoral cartilage degeneration. Arch Orthop Trauma Surg 2013;133(9):1295-1301

20 Jang SJ, Kim JD, Cha SS. Platelet-rich plasma (PRP) injections as an effective treatment for early osteoarthritis. Eur J Orthop Surg Traumatol 2013;23(5):573-580

21 Kon E, Mandelbaum B, Buda R, et al. Platelet-rich plasma intraarticular injection versus hyaluronic acid viscosupplementation as treatments for cartilage pathology: from early degeneration to osteoarthritis. Arthroscopy 2011;27(11):1490-1501

22 Filardo G, Kon E, Pereira Ruiz MT, et al. Platelet-rich plasma intraarticular injections for cartilage degeneration and osteoarthritis: single- versus double-spinning approach. Knee Surg Sports Traumatol Arthrosc 2012;20(10):2082-2091

23 Freitag J, Barnard A, Rotstein A. Photoactivated platelet-rich plasma therapy for a traumatic knee chondral lesion. BMJ Case Rep 2012;2012:pii: bcr2012006858

24 Sánchez M, Azofra J, Anitua E, et al. Plasma rich in growth factors to treat an articular cartilage avulsion: a case report. Med Sci Sports Exerc 2003;35(10):1648-1652

25 Guney A, Akar M, Karaman I, Oner M, Guney B. Clinical outcomes of platelet rich plasma (PRP) as an adjunct to microfracture surgery in osteochondral lesions of the talus. Knee Surg Sports Traumatol Arthrosc 2013; November 30 (Epub ahead of print); doi: 10.1007/ s00167-013-2784-5

26 Siclari A, Mascaro G, Gentili C, Kaps C, Cancedda R, Boux E. Cartilage repair in the knee with subchondral drilling augmented with a platelet-rich plasma-immersed polymer-based implant. Knee Surg Sports Traumatol Arthrosc 2014;22(6):1225-1234

27 Abrams GD, Mall NA, Fortier LA, Roller BL, Cole BJ. BioCartilage: background and operative technique. Oper Tech Sports Med 2013; 21:116-124

28 Lopez-Vidriero E, Goulding KA, Simon DA, Sanchez M, Johnson DH. The use of platelet-rich plasma in arthroscopy and sports medicine: optimizing the healing environment. Arthroscopy 2010; 26(2):269-278

29 Sadoghi P, Rosso C, Valderrabano V, Leithner A, Vavken P. The role of platelets in the treatment of Achilles tendon injuries. J Orthop Res 2013;31(1):111-118

30 Krogh TP, Bartels EM, Ellingsen T, et al. Comparative effectiveness of injection therapies in lateral epicondylitis: a systematic review and network meta-analysis of randomized controlled trials. Am J Sports Med 2013;41(6):1435-1446

31 Carter MJ, Fylling CP, Parnell LK. Use of platelet rich plasma gel on wound healing: a systematic review and meta-analysis. Eplasty 2011;11:e38

32 Villela DL, Santos VL. Evidence on the use of platelet-rich plasma for diabetic ulcer: a systematic review. Growth Factors 2010;28(2): 111-116

33 Braun HJ, Kim HJ, Chu CR, Dragoo JL. The effect of platelet-rich plasma formulations and blood products on human synoviocytes: implications for intra-articular injury and therapy. Am J Sports Med 2014;42(5):1204-1210

34 Dold AP, Zywiel MG, Taylor DW, Dwyer T, Theodoropoulos J. Platelet-rich plasma in the management of articular cartilage pathology: a systematic review. Clin J Sport Med 2014;24(1): 31-43

35 Filardo G, Kon E, Roffi A, Di Matteo B, Merli ML, Marcacci M. Platelet-rich plasma: why intra-articular? A systematic review of preclinical studies and clinical evidence on PRP for joint degeneration. Knee Surg Sports Traumatol Arthrosc 2013; November 26 (Epub ahead of print); doi: 10.1007/s00167-013-2743-1 